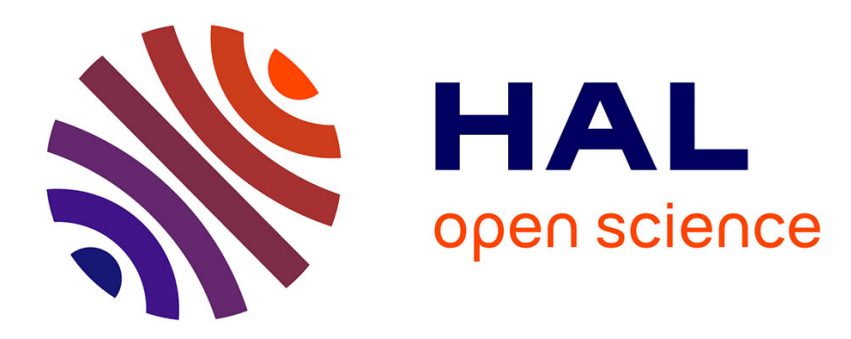

\title{
An experimental study of gelation of chains
}

\author{
F. Schosseler, L. Leibler
}

\section{To cite this version:}

F. Schosseler, L. Leibler. An experimental study of gelation of chains. Journal de Physique Lettres, 1984, 45 (10), pp.501-507. 10.1051/jphyslet:019840045010050100 . jpa-00232374

\section{HAL Id: jpa-00232374 https://hal.science/jpa-00232374}

Submitted on 1 Jan 1984

HAL is a multi-disciplinary open access archive for the deposit and dissemination of scientific research documents, whether they are published or not. The documents may come from teaching and research institutions in France or abroad, or from public or private research centers.
L'archive ouverte pluridisciplinaire HAL, est destinée au dépôt et à la diffusion de documents scientifiques de niveau recherche, publiés ou non, émanant des établissements d'enseignement et de recherche français ou étrangers, des laboratoires publics ou privés. 
Classification

Physics Abstracts

$36.20 \mathrm{E}-82.70 \mathrm{G}-82.50$

\title{
An experimental study of gelation of chains
}

\author{
F. Schosseler and L. Leibler \\ Centre de Recherches sur les Macromolécules, CNRS, \\ 6, rue Boussingault, 67083 Strasbourg Cedex, France
}

(Reçu le 24 février 1984, accepté le 26 mars 1984)

\begin{abstract}
Résumé. - Nous étudions la gélification par rayonnement gamma d'une solution de polystyrène et de cyclopentane. La réaction est arrêtée à différentes étapes et le sol est caractérisé par diffusion de lumière après dilution en bon solvant. Nous obtenons la variation du rayon de gyration moyen des polymères en fonction de leur masse moyenne. Elle semble montrer que la gélification de chaînes courtes au voisinage de leur concentration de recouvrement est décrite plutôt par des exposants de percolation que par des exposants classiques.
\end{abstract}

\begin{abstract}
Gamma-radiation induced gelation of polystyrene solution in cyclopentane is studied. The reaction is stopped at different stages and the properties of the diluted sol are characterized by elastic light scattering. We have measured the dependence of the average radius of gyration as a function of the average molecular weight of extracted polymers. It seems to indicate that in the case of relatively short chains and concentration close to the overlap concentration the gelation is characterized by percolation rather than classical exponents.
\end{abstract}

\section{Introduction.}

A very important effort has been devoted to theoretical studies of the gelation processes [1]. This includes classical Flory [2] and Stockmayer [3] theories and their extension the cascade model $[4,5]$, as well as recent percolation [6,7] and kinetic models [8]. These theories lead to qualitatively different predictions concerning the properties of the sol-gel transition. In the case of the gelation of chains the classical or percolation behaviour are expected to be observed depending on the molecular weight of the chains and on the solution concentration $[9,10]$. Experimentally however, little is known about the gelation of chains.

The irradiation of polystyrene solutions in cyclopentane by $\gamma$-rays leads to the formation of gels [11]. We have undertaken systematic studies of this gelation process. The system presents two major advantages for the study of the sol-gel transition. First, it is not necessary to make instantaneous measurements in situ. We can stop the irradiation at any moment, perform the measurements and then continue the irradiation and the reaction. Secondly, the advancement of the reaction is proportional to the radiation dose and is easy to control through the exposure time.

In order to study the variation of sol properties during this gelation process we have stopped the reaction at different stages and diluted the polymers extracted from the sol in a good solvent. 
Daoud et al. [12] have pointed out recently, that the studies of physico-chemical properties of the diluted sol as a function of the average molecular weight of extracted polymers provide a measure of the polydispersity of molecules and, therefore, may be useful as a test of validity of different models of gelation. The advantage of such a procedure is that it does not require the knowledge of the exact advancement of the reaction for each particular sample. In this note we report the variation of the average radius of gyration as a function of the molecular weight obtained by elastic light scattering.

\section{Experimental.}

We prepared solutions of linear polystyrene with molecular weight $M_{\mathrm{w}}=55000$ and with polydispersity $M_{\mathrm{w}} / M_{\mathrm{n}}=1.2$ in cyclopentane at a concentration $c=0.1 \mathrm{~g} / \mathrm{cm}^{3}$. After careful degassing the solutions were irradiated with $\gamma$-rays. A gel is formed after about $10^{5} \mathrm{~min}$. The irradiation was stopped at different times ranging from $4.3 \times 10^{4}$ to $9.6 \times 10^{4} \mathrm{~min}$. Then the sol was washed in toluene, lyophilized in benzene and dried under vacuum at $60{ }^{\circ} \mathrm{C}$.

The polymers that we have extracted were then diluted in toluene and elastic light scattering measurements with a Sofica $50(\lambda=436 \mathrm{~nm}$ or $\lambda=546 \mathrm{~nm})$ or a Sofica $4200(\lambda=632 \mathrm{~nm})$ photogoniometer were performed. We used scattering angles between $15^{\circ}$ and $150^{\circ}$. Blue light $(\lambda=436 \mathrm{~nm})$ was used for the lowest molecular weights and green $(\lambda=546 \mathrm{~nm})$ or red $(\hat{\lambda}=$ $632 \mathrm{~nm}$ ) for more irradiated samples. However, for samples close to the gel point it is not possible to work in the Guinier range of the scattering wave vector $q$ which is much smaller than the inverse of the radius of gyration. For these samples Zimm representation of the scattered intensity presents a strong curvature. If one neglects this curvature and uses a straight line extrapolation to zero angle for determining the average molecular weight $\boldsymbol{M}_{\mathrm{w}}$ and the average radius of gyration $\left\langle R_{\mathrm{G}}^{2}\right\rangle_{z}$ only a rough estimation of these quantities is obtained. Actually a more complete analysis is needed since higher moments of the molecular weight distribution do contribute to the scattered intensity. We used then a more complete expression for the scattered intensity $I(q)$ :

$$
\frac{K c}{I(q)}=\frac{1}{M_{\mathrm{w}}}\left[1+\frac{q^{2}}{3}\left\langle R_{\mathrm{G}}^{2}\right\rangle_{z}-\frac{q^{4}}{12}\left(\left\langle\rho^{4}\right\rangle_{z}-\frac{4}{3}\left\langle R_{\mathrm{G}}^{2}\right\rangle_{z}\right)\right]+2 A_{2} c
$$

where $K$ denotes an apparatus constant, $c$ is the monomer concentration and $A_{2}$ is the second virial coefficient. The $z$-average $\langle A\rangle_{z}$ appearing in (1) is defined as :

$$
\langle A\rangle_{z}=\frac{\int A(N) N^{2} P(N) \mathrm{d} N}{\int N^{2} P(N) \mathrm{d} N}
$$

where $P(N)$ is the number of molecules with $N$ monomer units. The radius of gyration $R_{\mathrm{G}}^{2}$ and the fourth moment $\rho^{4}$ are defined for each molecule by :

$$
\begin{aligned}
R_{\mathrm{G}}^{2} & =\left\langle\frac{1}{2 N^{2}} \sum_{i=1}^{N} \sum_{j=1}^{N} r_{i j}^{2}\right\rangle_{T} \\
\rho^{4} & =\left\langle\frac{1}{10 N^{2}} \sum_{i=1}^{N} \sum_{j=1}^{N} r_{i j}^{4}\right\rangle_{T}
\end{aligned}
$$

where $r_{i j}$ is the distance between monomers $i$ and $j$ and $\langle\quad\rangle_{T}$ denotes the thermal average over all configurations of the molecules in the solvent. For critical objects with only one characteristic length (e.g. linear polymers with excluded volume) $R_{G}^{2}$ and $\rho^{4}$ are related by a simple relation. 
In our case we treat $\left\langle R_{G}^{2}\right\rangle_{z}$ and $\left\langle\rho^{4}\right\rangle_{z}$ as independent fitting parameters. Such a procedure has been already found useful to characterize branched polycondensates and high molecular weight linear chains $[13,14]$.

Figure 1 shows a typical Zimm-plot for a highly branched and polydisperse sample irradiated during $8.74 \times 10^{4} \mathrm{~min}$. The experimental data can be well fitted with expression (1) as is illustrated in figure 2 . The errors are typically of a few per cent which is much the same as the experimental deviation of $c / l$. The numerical fit gives the molecular weight and the radius of gyration equal to $M_{\mathrm{w}}=1.84 \times 10^{6}$ and $\left\langle R_{\mathrm{G}}^{2}\right\rangle_{\mathrm{z}}^{1 / 2}=712 \AA$, respectively. These values are to be compared with a classical linear extrapolation which keeps all values of scattering angles and gives $M_{\mathrm{w}}=$ $1.75 \times 10^{6}$ and $\left\langle R_{\mathrm{G}}^{2}\right\rangle^{1 / 2}=640 \AA$. Better values, $M_{\mathrm{w}}=1.83 \times 10^{6}$ and $\left\langle R_{\mathrm{G}}^{2}\right\rangle^{1 / 2}=701 \AA$ are obtained if only the smallest values of scattering angles are used. However when more irradiated samples are studied such an extrapolation overestimates largely the molecular weight.

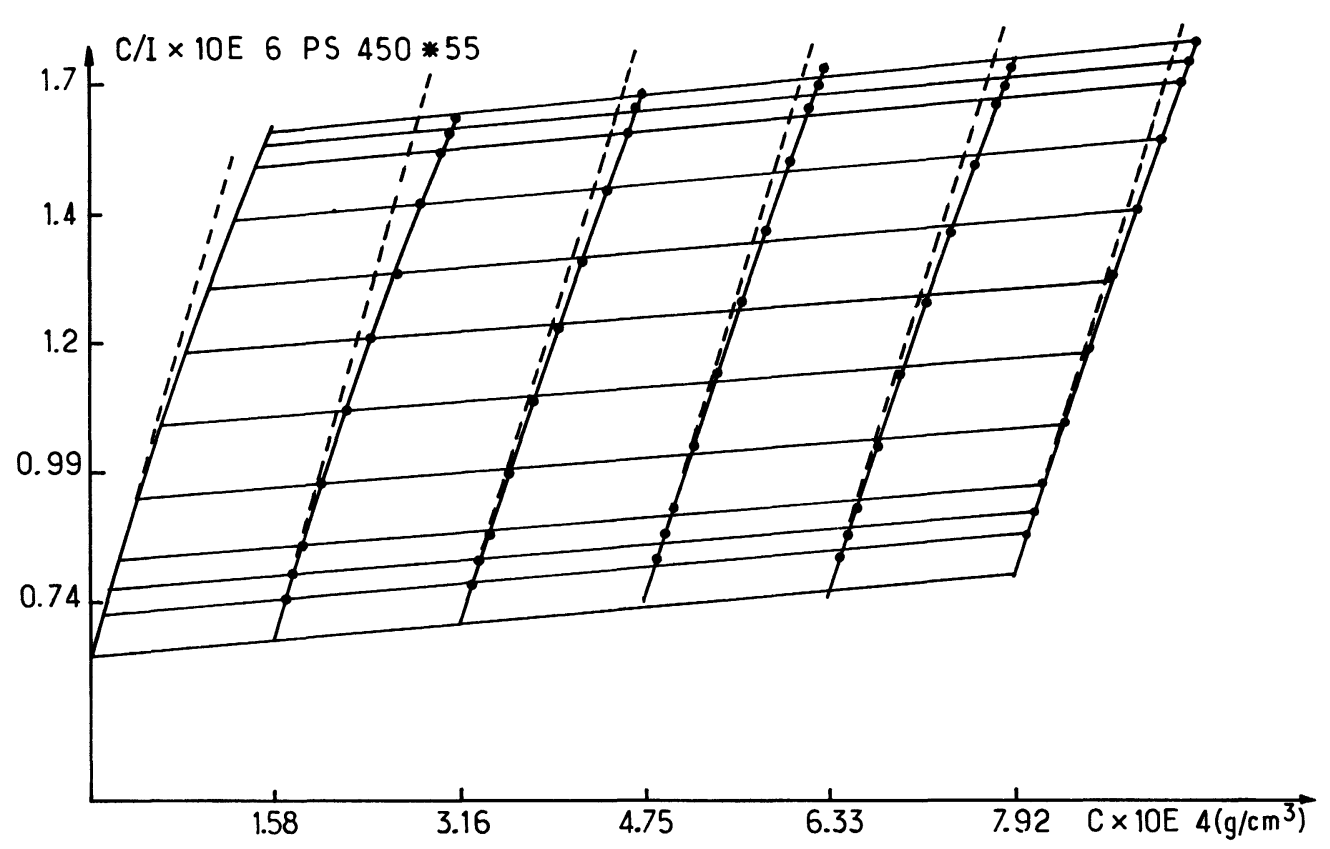

Fig. 1. - A typical Zimm-plot $c / I$ vs. $K c+\sin ^{2}(\theta / 2)$ where $\theta$ is the scattering angle. This sample irradiated during $87400 \mathrm{~min}$ exhibits already a curvature. Continuous lines are obtained by fitting the data with equation (1). One finds $M_{\mathrm{w}}=1.84 \times 10^{6},\left\langle R_{\mathrm{G}}^{2}\right\rangle_{z}^{1 / 2}=712 \AA,\left\langle\rho^{4}\right\rangle^{1 / 2}=401 \AA$. Dashed lines represent linear extrapolation through smallest values of scattering angles. In this particular case the two procedures lead to the same result for $M_{\mathrm{w}}$ and $\left\langle R_{\mathrm{G}}^{2}\right\rangle_{z}$.

The fourth moment $\left\langle\dot{\rho}^{4}\right\rangle_{z}$ can be determined precisely only for the most irradiated samples. We find that the ratio $\left\langle R_{G}^{2}\right\rangle /\left\langle\rho^{4}\right\rangle$ is about 0.7 .

In figure 3 we have plotted the dependence of the radius of gyration of branched polymers as a function of their molecular weight. These data may be fitted by the empirical relationship :

$$
\left\langle R_{\mathrm{G}}^{2}\right\rangle_{z}=0.024 M_{\mathrm{w}}^{1.16} \AA^{2}
$$

with an accuracy of about ten per cent on the exponent. 


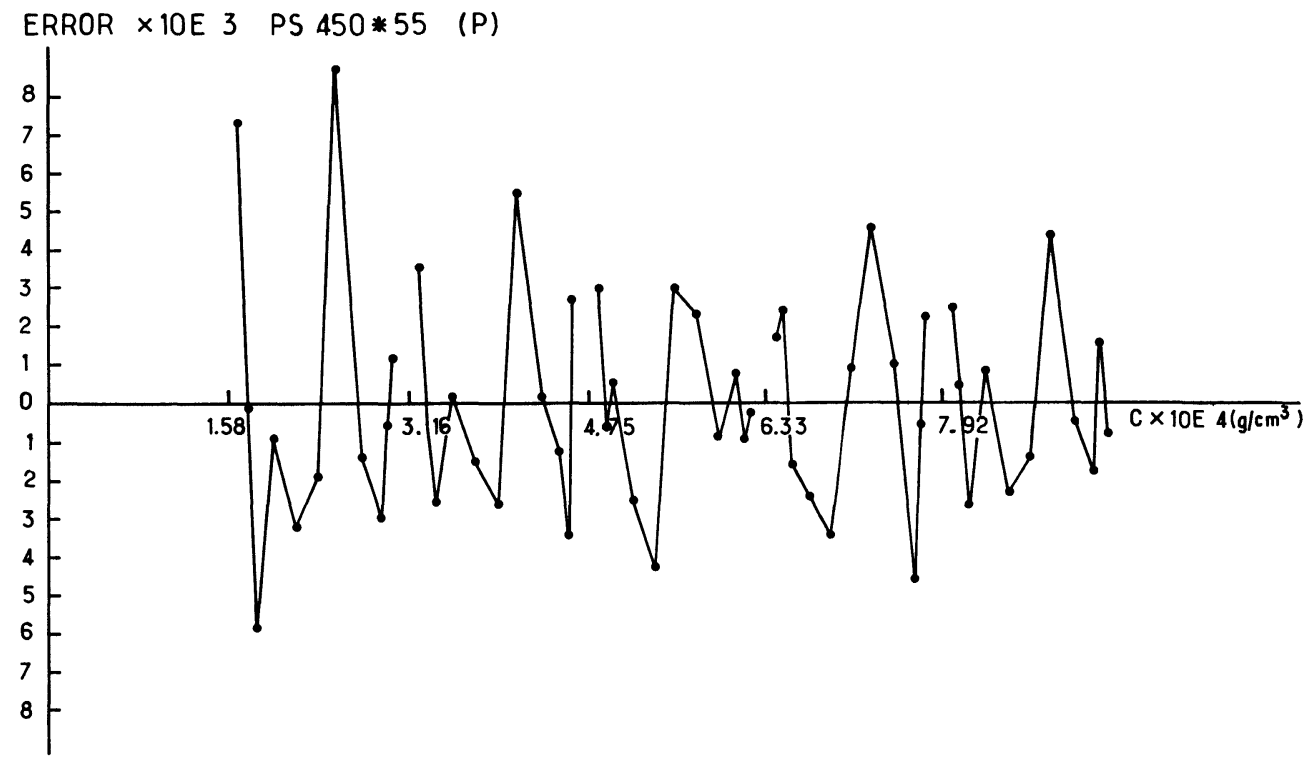

Fig. 2. - Plot of the relative error of the fit $\left((c / I)_{\exp }-(c / I)_{\mathrm{th}}\right) /(c / I)_{\exp }$ for the same system as in figure 1 . The $x$-scale is the same as in figure 1. The greatest deviation in this case appears to be less than one per cent. Moreover no correlation can be detected in the deviations.

\section{Discussion.}

The interpretation of the above results requires a clear distinction between two stages of the experiment (cf. Ref. [12]). First, during the irradiation process linear chains crosslink and randomly branched molecules are formed. When the irradiation is stopped, certain distribution of molecular weights is obtained. The number of the $N$-mers $P(N)$ depends both on $N$ and the advancement of the reaction. The distribution $P(N)$ determines the average molecular weight $M_{\mathrm{w}}$. Secondly, the sol is diluted and the molecules swell. As pointed out by Daoud et al. [12] the measured radius of gyration depends both on the swelling of each molecule and the distribution $P(N)$.

In the case of gelation of chains the distribution $P(N)$ depends on the polymerization index, $N_{0}$, of the precursor chains and the solution concentration $[9,10]$. When the primary chains are long and/or the solution is very concentrated the distribution is expected to be of the mean field type except for an extremely narrow critical region near the gel point. The distribution $P(N)$ has been then calculated by Stockmayer [3] and can be written in the following scaling form near the gel point

$$
P(N) \sim N_{0}\left(N / N_{0}\right)^{-5 / 2} \exp \left\{-A N \varepsilon^{2} / N_{0}\right\}
$$

where $\varepsilon=\left(p_{\mathrm{c}}-p\right) / p_{\mathrm{c}}$ is the relative distance from the gel point with $p$ characterizing the advancement of the reaction. $A$ depends on both the concentration and the length of precursor chains.

In the case of shorter chains or relatively dilute solutions the critical region is larger and a percolation distribution should be observable. The natural generalization of (6) is to write

$$
P(n) \sim N_{0} n^{-\tau} f\left(\varepsilon n^{\sigma}\right)
$$




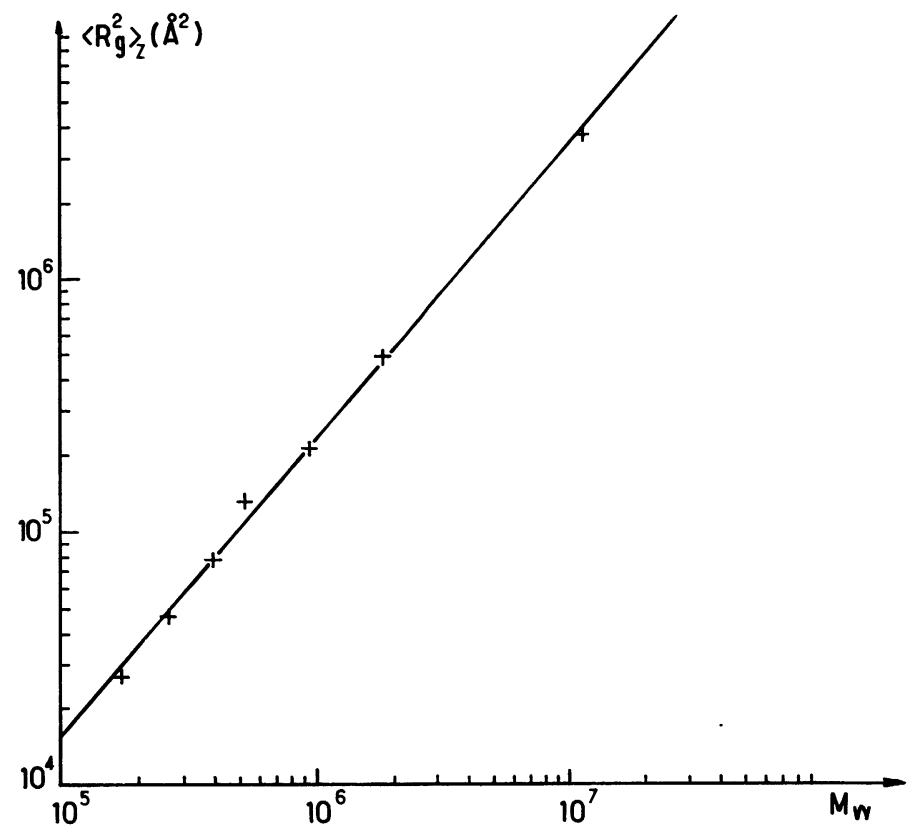

Fig. 3. - Double logarithmic plot of $\left\langle R_{\mathrm{G}}^{2}\right\rangle_{z} v s$. $M_{\mathrm{w}}$. The straight line corresponds to the experimental law $\left\langle R_{\mathrm{G}}^{2}\right\rangle_{z}=0.024 . M^{1.16} \AA^{2}$ obtained by least squares adjustment. Crosses correspond to an experimental error of $\pm 5 \%$ in determining $M_{\mathrm{w}}$ and $\left\langle R_{\mathrm{G}}^{2}\right\rangle_{z}$.

where $n=N / N_{0}$ denotes the number of primary chains in the branched molecule and $f$ is a universal function. $\tau$ and $\sigma$ are percolation exponents rather than the classical ones $\tau_{\mathrm{cl}}=5 / 2$ and $\sigma_{\mathrm{cl}}=1 / 2$. From (7) the average molecular weight varies like

$$
M_{\mathrm{w}} \sim N_{0} \varepsilon^{-\gamma}
$$

where exponent $\gamma=(3-\tau) / \sigma$. In the percolation theory $\gamma \sim 1.7$ at three dimensions.

If one assumes that after the dilution in a good solvent all $N$-mers swell in the same way characterized by the exponent $v$ (i.e. $R_{G}^{2} \sim N^{2 v}$ ) one gets from (7) (cf. Ref. [12]) :

$$
\left\langle R_{\mathrm{G}}^{2}\right\rangle_{z}=\frac{\int N^{2 v} n^{2} P(n) \mathrm{d} n}{\int n^{2} P(n) \mathrm{d} n} \sim N_{0}^{2 v} \varepsilon^{-2 v / \sigma} .
$$

Hence, combining equations (8) and (9) yields the variation of $\left\langle R_{\mathrm{G}}^{2}\right\rangle_{\mathbf{z}}$ as a function of $M_{\mathrm{w}}$ :

$$
\left\langle R_{\mathrm{G}}^{2}\right\rangle_{z} \sim M_{\mathrm{w}}^{2 v_{\text {eff }}} N_{0}^{-2 v \beta / \gamma}
$$

with

$$
v_{\text {eff }}=(1+\beta / \gamma) v
$$

and $\beta=(\tau-2) / \sigma$. (In the percolation theory $\beta \sim 0.34(d=3)$.)

It is to be noted that when chains with the same average molecular weight are compared the 
clusters made up from shorter precursor should have bigger average radius of gyration. If one takes for $v$ the exponent obtained for randomly branched chains without loops [15], $v=0.5$, one gets $v_{\text {eff }}=1$ if a classical behaviour is observed and $v_{\text {eff }} \simeq 0.63$ with the percolation exponents [12].

It should be stressed that the assumption that all chains swell in the same manner is not very realistic for the gelation of chains. Even near the gelation point an important fraction of noncrosslinked chains remains and they swell more than branched molecules when diluted. Nevertheless, in the case of short chains which we have used in the experiment, their contribution to the wave vector dependent part of the scattering intensity is very small hence the largest contribution to the radius of gyration that we measure comes from branched chains with high molecular weights. Therefore, equation (11) should be essentially correct in our case.

Equation (10) holds only if chains are highly branched. If we suppose a limit case where only a few crosslinks are present on a primary chain, we can introduce the length of the primary chain as the elementary unit through

$$
R_{\mathrm{G}}(N) \simeq\left(\frac{N}{N_{0}}\right)^{v} N_{0}^{v_{\mathrm{L}}} a
$$

where $v_{\mathrm{L}}$ characterizes the swelling of linear chains and $a$ is the monomer length. In this particular case the formula takes correctly into account the swelling of linear chains present in the system

$$
\left\langle R_{\mathrm{G}}^{2}\right\rangle_{z} \sim M_{\mathrm{w}}^{2 v_{\text {eff }}} N_{0}^{2\left(v_{\mathrm{L}}-v_{\text {eff }}\right)}
$$

instead of (10) [16]. The molecular weight dependence of the radius of gyration remains the same. The dependence on the primary chains length becomes weaker $\left(N_{0}^{-0.06}\right.$ instead of $\left.N_{0}^{-0.26}\right)$.

The exponent which we find experimentally $v_{\text {eff }} \simeq 0.58$ (cf. Eq. (5)) would then indicate that the gelation is described by percolation rather than classical exponents. This might be expected for the gelation of short chains and at a concentration close to the overlap concentration $c^{*}$ which we have studied here.

In view of the present results it would be interesting to study the effect of molecular weight of precursors and of their concentration on the gelation processes.

\section{Acknowledgments.}

We are deeply indebted to J. Bastide and J. Marchal, who shared with us their knowledge of preparing gels by $\gamma$-irradiation, for their continuous help and interest. We gratefully acknowledge interesting discussions with H. Benoît, J. Candau, M. Daoud, P. G. de Gennes, J. F. Joanny, J. P. Munch and C. Strazielle. We would like to thank A. Lapp who kindly synthesized polystyrene chains for us.

\section{References}

[1] See for example the review article :

Stauffer, D., Coniglio, A. and Adam, M., Adv. Polym. Sci. 44 (1982) 103 and references therein.

[2] Flory, P. J., Principles of polymer chemistry (Cornell University Press, Ithaca, N.Y.) 1953, Chap. IX.

[3] Stockmayer, W. H., J. Chem. Phys. 12 (1944) 125.

[4] Gordon, M., Proc. R. Soc. A 268 (1962) 240.

Good, I. J., Proc. R. Soc. A 272 (1963) 54.

[5] Burchard, W., Adv. Polym. Sci. 48 (1983) and references therein.

[6] De Gennes, P. G., J. Physique Lett. 37 (1976) L-1.

[7] STAuffer, D., J. Chem. Soc. Faraday Trans. 272 (1976) 1354. 
[8] ZifF, R. M., Hendriks, E. M., ERnst, M. H., Phys. Rev. Lett.43(1982) 593.

Manneville, P., De Sèze, L., in Numerical Methods in the Study of Critical Phenomena, Edited by J. Della Dora, J. Demongeot and B. Lacolle (Springer Verlag. Berlin) 1981.

Herrmann, H. J., Landau, D. P., Stauffer, D., Phys. Rev. Lett. 49 (1983) 412.

[9] De Gennes, P. G., J. Physique Lett. 38 (1977) L-355.

[10] Daoud, M., J. Physique Lett. 40 (1979) L-201.

[11] BASTIDE, J., Thèse d'Etat, Université L. Pasteur, Strasbourg. 1984, available on request.

[12] DaOud, M., Family, F., JanninK, G., J. Physique Lett. 45 (1984) L-199.

[13] Gordon, M., Kajiwara, K., Peniche-Covas, C. A. L. and Ross-Murphy, S. B., Makromol. Chem. 176 (1975) 2413.

[14] Shinbo, K., Miyake, Y., J. Phys. Soc. Jpn 48 (1980) 2084.

[15] IsaAcson, J., Lubensky, T. C., J. Physique Lett. 41 (1980) L-463.

Daoud, M., Joanny, J. F., J. Physique Lett. 42 (1981) 1359.

[16] DAOUD, M., private communication. 